\title{
Electrokinetic flow in connected channels: a comparison of two circuit models
}

\author{
Christian J. C. Biscombe · Malcolm R. Davidson · \\ Dalton J. E. Harvie
}

Received: [date] / Accepted: [date] / Published online: [date]

\begin{abstract}
We apply the recently developed 'ion current model' (ICM) to investigate mixed pressure-driven/electro-osmotic flow in electrokinetic series circuits. Under the ICM, the local geometric mean ion concentration varies throughout a microfluidic circuit as a consequence of ion conservation. ICM predictions are used to assess the accuracy of the earlier 'total current model' (TCM), which is instead based upon the simplifying assumption that the geometric mean ion concentration is uniform in all channels within a circuit. The model comparison is performed over an experimentally relevant range of physical parameters and channel dimensions. When the ratio of total current to flow rate is small, or when the inlet concentration is very low or very high, the ICM and TCM give similar predictions of the total pressure and potential differences across simple three-channel contraction-expansion networks. Otherwise, the errors introduced by using the TCM may be very large. Our results demonstrate that the common assumption that the electrolyte concentration throughout a device is equal to the concentration of fluid supplied to the device is not always valid, even if there is little or no electric double layer overlap.
\end{abstract}

Keywords Electrokinetic $\cdot$ Microfluidic $\cdot$ Circuit $\cdot$ Contraction

Electronic supplementary material The online version of this article (doi:[doi]) contains supplementary material, which is available to authorized users.

Christian J. C. Biscombe · Malcolm R. Davidson · Dalton J. E. Harvie

Department of Chemical and Biomolecular Engineering, The University of Melbourne, Parkville, VIC 3010, Australia

E-mail: daltonh@unimelb.edu.au 


\section{Introduction}

The increasing complexity of microfluidic (or lab-on-a-chip) devices is expected to drive demand for computational tools, such as electrokinetic circuit models, to aid device design and optimisation (Erickson 2005; Boy et al. 2008). One-dimensional circuit models (such as those to be considered herein) are used to predict electrokinetic flow behaviour in microchannel networks by relating the driving forces (pressure difference $\Delta p$ and electrical potential difference $\Delta \phi$ ) to their conjugate transport rates (volumetric flow rate $Q$ and current $I$ ) in terms of known (or measurable) physicochemical properties (Wachutka 1995; Ajdari 2004).

Electrokinetically driven flows are employed for many applications in chemistry and biotechnology, including DNA and protein/peptide analyses, immunoassays, and enzyme assays (Bousse et al. 2000; Bruin 2000; Verpoorte 2002). Electrokinetic phenomena develop in response to the electrostatic charge acquired by most solid surfaces when brought into contact with an electrolyte solution (Hunter 1981). The surface charge promotes a redistribution of ions within the liquid, leading to the formation of a charged electric double layer (EDL) adjacent to the interface. For fully developed flow of symmetric, binary, Newtonian electrolytes in slit or cylindrical channels, the transverse cation $(+)$ and anion $(-)$ concentration profiles within the diffuse part of the EDL obey Boltzmann distributions (Harvie et al. 2012):

$$
n_{ \pm}=n_{0} \exp \left(-\frac{z_{ \pm} e}{k T} \psi\right)
$$

where $z_{ \pm}$are the ion valencies, $e$ is the elementary charge, $k$ is the Boltzmann constant, $T$ is the absolute temperature, and $\psi$ is the EDL potential. Eq. (1) is valid within any uniform channel, regardless of the degree of EDL overlap, provided that the reference ion concentration $n_{0}$ is taken to be the geometric mean ion concentration at any position within that channel (that is, $n_{0}=\sqrt{n_{+} n_{-}}$) (Harvie et al. 2012).

Electrokinetic circuit models consist of two parts: (i) flow equations for each channel in the network, and (ii) conservation equations for circuit nodes (channel junctions). End effects local to nodes are neglected, so that the flow is regarded as being fully developed everywhere. Consequently, the combined liquid-solid system is electroneutral at every axial location.

The flow equations describing fully developed, laminar, steady state, electrokinetic flow are

$$
\left[\begin{array}{l}
Q \\
I
\end{array}\right]=\left[\begin{array}{ll}
L_{11} & L_{12} \\
L_{21} & L_{22}
\end{array}\right]\left[\begin{array}{l}
\Delta p \\
\Delta \phi
\end{array}\right] .
$$


This formulation can be viewed as an extension of Ohm's law from electronics (Ajdari 2004), with each channel contributing discrete hydrodynamic and electrical resistances. The conductances $L_{i j}(i, j \in[1,2]$; see the Appendix for definitions) depend on the channel geometry (shape and dimensions) and electrolyte properties (liquid viscosity $\mu$ and dielectric constant $\varepsilon_{\mathrm{f}}$, as well as ion valencies $z_{ \pm}$and diffusivities $D_{ \pm}$); all except $L_{11}$ also depend on the temperature $T$, ionic concentration $n_{0}$, and surface charge density $\sigma_{\mathrm{d}}$ (or alternatively zeta potential $\zeta$ ). The conductances exhibit Onsager reciprocity (i.e. $L_{12}=L_{21}$ ) (Onsager 1931a,b) irrespective of the cross-sectional geometry of the channels (Sørensen and Koefoed 1974; Brunet and Ajdari 2004).

Conservation laws are required at circuit nodes to link together the fully developed flow solutions within each of the discrete 'resistors' (channels). Volume and total charge must be conserved across nodes:

$$
\begin{aligned}
& \sum_{m=1}^{M} Q^{(m)}=0, \\
& \sum_{m=1}^{M} I^{(m)}=0,
\end{aligned}
$$

where $M$ is the number of channels that intersect at the node, and $Q^{(m)}$ and $I^{(m)}$ are the flow rate and total current respectively in branch $m$, with flow towards the node defined as positive (Ajdari 2004; Xuan and Li 2004; Harvie et al. 2012). These equations are analogous to Kirchhoff's current law from electronics. Herein we consider only serially connected channels, so $Q$ and $I$ are both uniform throughout all channels. An additional nodal constraint on ion species is also required. Two possibilities have been proposed:

1. Total current model (TCM). For a stationary system (i.e. no net fluxes), axial equilibrium is achieved and the geometric mean ion concentration $n_{0}$ within any channel $m$ is equal to its value at the inlet to the network:

$$
n_{0}^{(m)}=n_{0}^{(\mathrm{in})} .
$$

For a non-stationary system, Eq. (5) is not necessarily valid (Biscombe et al. 2012), but it has nevertheless been used by several authors in their descriptions of electrokinetic flow systems (Qiao and Aluru 2002; Xuan and Li 2004; Berli 2007, 2008). The resultant 'total current model' (TCM), which comprises Eqs. (2), (3), (4), and (5), is linear and may be solved straightforwardly when the flow rate and total current are specified (as in the cases to be considered herein). Once $n_{0}^{(\text {in })}$ is chosen, the conductances for every 
channel can be calculated immediately (by making use of Eq. (5)) and the pressure and potential changes can be determined from Eq. (2) using (e.g.) Cramer's rule (Xuan and Li 2004; Berli 2007). Our implementation of this model is equivalent to those of Xuan and $\operatorname{Li}(2004)$ and Berli $(2007,2008)$ except that we allow the cations and anions to have unequal diffusivities.

2. Ion current model (ICM). Alternatively, we proposed to use the more fundamental condition that the ion currents $I_{ \pm}$, related to the total current by $I=I_{+}+I_{-}$, must be conserved at nodes (Harvie et al. 2012; Biscombe et al. 2012):

$$
\sum_{m=1}^{M} I_{ \pm}^{(m)}=0
$$

Eq. (6) is valid whether or not the system is stationary. The 'ion current model' (ICM) comprises Eq. (2), (3), and (6) (Eq. (4) is automatically satisfied if Eq. (6) is satisfied). Unlike the TCM, the value of $n_{0}$ in each channel must be chosen such that Eq. (6) is satisfied at each node, which has two important implications. Firstly, the reference ion concentrations for each channel need not be equal (i.e. Eq. (5) does not necessarily hold). Secondly, since $L_{12}, L_{21}, L_{22}$, and $I_{ \pm}$are all functions of $n_{0}$, the ICM is non-linear and requires an iterative numerical solution procedure.

Due to the different nodal species constraints used by the TCM and ICM (Eqs. (5) and (6) respectively), the set of conductances for each of the two models may differ, which may in turn lead to differences between predictions for $\Delta p$ and $\Delta \phi$ (from Eq. (2)). It would be desirable to use the TCM whenever possible, because of its relative simplicity, but the use of Eq. (5) for non-stationary systems has not been rigorously assessed. We recently showed that for the special case of electroviscous flow (i.e. $I=0)$ through a particular contractionexpansion network, TCM predictions of the total pressure and potential differences are in error by less than $3 \%$ and $17 \%$ respectively, compared to the ICM (Biscombe et al. 2011). Our aim in this paper is to elucidate the specific circumstances under which the TCM is a useful approximation for the more general case of mixed pressure-driven/electro-osmotic flow $(I \neq 0)$, over a realistic range of physical parameters and channel dimensions. Again we consider a contraction-expansion, since this geometry is directly relevant to the microfluidics and membrane science communities and has been widely investigated in both experimental and numerical contexts (Pu et al. 2004; Plecis et al. 2005; Wang et al. 2005; Park et al. 2006; Ramirez and Conlisk 2006; Davidson and Harvie 2007; Kim et al. 2007; Bharti et al. 2008; 
Hughes et al. 2008; Postler et al. 2008; Mani et al. 2009; Wang et al. 2009; Zangle et al. 2009; Berry et al. 2011; Biscombe et al. 2012).

\section{Problem specification}

For our 'base case' we consider a 100:1:100 cylindrical contraction-expansion network (see Fig. 1), chosen for consistency with our earlier work on electroviscous flow (Biscombe et al. 2011). The inlet and outlet channels are large relative to the contraction and effectively function as reservoirs; in this sense our channel arrangement is similar to that used in typical electrokinetic experiments (Stein et al. 2004; van der Heyden et al. 2005).

Model performance is evaluated by specifying the flow rate $Q$, total current $I$, and inlet concentration $n_{0}^{\text {(in) }}$, and then comparing TCM and ICM predictions of the total pressure and potential differences, $\Delta p_{\mathrm{t}}=\Delta p^{(\mathrm{in})}+\Delta p^{(\mathrm{con})}+\Delta p^{(\mathrm{out})}$ and $\Delta \phi_{\mathrm{t}}=\Delta \phi^{(\mathrm{in})}+\Delta \phi^{(\mathrm{con})}+$ $\Delta \phi^{\text {(out) }}$ respectively, occurring along the entire domain. It is recognised that local to nodes, where the cross-sectional geometry changes, entrance and exit effects (e.g. concentration polarisation) will contribute additional node-based terms to $\Delta p_{\mathrm{t}}$ and $\Delta \phi_{\mathrm{t}}$. We neglect these extra contributions by assuming that every channel within the circuit is sufficiently long that the fully developed flow contributions are generally of greater magnitude than the nodal terms.

We require the direction of the total current to be opposite to that of the net fluid flow (i.e. $I / Q<0$ ), which guarantees that (i) the ICM has a unique solution (Biscombe et al. 2012) (non-uniqueness would hinder direct comparison with the TCM, which always has a unique solution); and (ii) strong concentration shocks (which arise when axial concentration characteristics collide (Mani et al. 2009)) will not occur within the network (see Eq. (29) of Biscombe et al. (2012)). In order to keep $I / Q<0$, the hydrodynamic and electro-osmotic components of the fluid flow must oppose each other (i.e. $\Delta p_{\mathrm{t}}<0$ and $\Delta \phi_{\mathrm{t}}>0$ ). Such opposing flows have been used to generate recirculation in contraction-expansion devices for particle separation ('flow-induced electrokinetic trapping' (Jellema et al. 2009, 2010)) and preconcentration (Lettieri et al. 2003).

Inlet concentrations ranging from $10^{-6} \mathrm{M}$ to $1 \mathrm{M}$ are considered. All channels are assumed to possess a uniform surface charge density $\sigma_{\mathrm{d}}$; for our base case we choose $\sigma_{\mathrm{d}}=-25 \mathrm{mCm}^{-2}$, which is typical of silica microchannels (Schoch and Renaud 2005; 


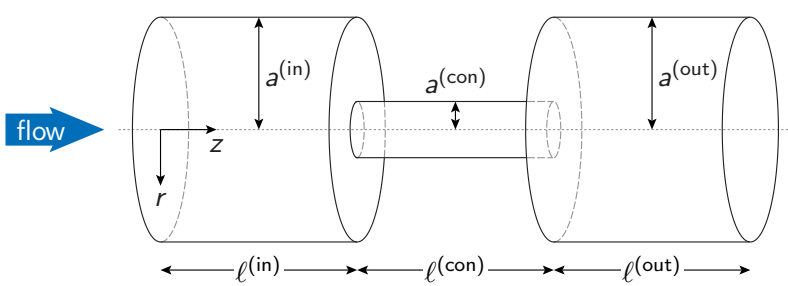

Fig. 1 Schematic diagram of the contraction-expansion flow geometry (not to scale). Dimensions are given in Table 1.

Table 1 Physical parameters and channel dimensions employed in this study.

\begin{tabular}{lc}
\hline Property & Value \\
\hline$T(\mathrm{~K})$ & 298.15 \\
$\mu\left(10^{-4} \mathrm{~kg} \mathrm{~m}^{-1} \mathrm{~s}^{-1}\right)$ & $8.9002^{a, b}$ \\
$\varepsilon_{\mathrm{f}}($ dimensionless $)$ & $78.408^{a, b}$ \\
$D_{+}\left(10^{-9} \mathrm{~m}^{2} \mathrm{~s}^{-1}\right)$ & $1.957^{a, c}$ \\
$D_{-}\left(10^{-9} \mathrm{~m}^{2} \mathrm{~s}^{-1}\right)$ & $2.032^{a, c}$ \\
\hline$a^{(\text {in })}=a^{(\text {out })}(\mu \mathrm{m})$ & 5.0 \\
$a^{(\text {con })}(\mu \mathrm{m})$ & 0.050 \\
$l^{\text {(in })}=l^{(\text {con })}=l^{(\text {out })}(\mu \mathrm{m})$ & 5000.0 \\
\hline
\end{tabular}

${ }^{a}$ Data from Haynes (2011)

${ }^{b}$ Based on properties of water at $25^{\circ} \mathrm{C}$ and

$0.1 \mathrm{MPa}$

${ }^{c}$ At infinite dilution in water at $25^{\circ} \mathrm{C}$

Andersen et al. 2011). Potassium chloride $(\mathrm{KCl})$ solution is selected as the electrolyte $\left(z_{+}=-z_{-}=z=1\right)$. Values of relevant physical parameters are listed in Table 1.

We systematically compare the TCM and ICM by examining the following variations on the base case (all other conditions being equal):

-switching to thin Cartesian slit (parallel plate) geometry;

-changing the contraction radius, keeping the flux ratio $-I / Q$ fixed at $10^{9} \mathrm{Cm}^{-3}$ (this corresponds, for instance, to a total current of $-10^{-10} \mathrm{~A}$ at a flow rate of $10^{-19} \mathrm{~m}^{3} \mathrm{~s}^{-1}$ ); -varying the surface charge density, keeping $-I / Q$ fixed as above; and

-altering the ionic diffusivities (see Electronic Supplementary Material; in summary we find that the trends described below are representative of a wide range of binary electrolytes). 
The conductances for both models are calculated using a finite volume numerical method (Harvie et al. 2012). A non-uniform mesh is used with 1,000 cells spanning the radius (or half-width, as appropriate) of each channel. We have confirmed that our results are practically insensitive to further mesh refinement.

We make a number of simplifying assumptions. The electrolyte is treated as a continuum with spatially uniform physical properties; changes in these properties due to changes in solute concentration are ignored. The surface charge density is treated as an intrinsic property of the solid wall material, neglecting possible variations driven by chemical equilibria in solution (Ninham and Parsegian 1971; Behrens and Grier 2001). Ion size effects, which can become important at high surface charge densities and high concentrations (Gillespie et al. 2011), are also neglected. Attention is restricted to univalent, symmetric, binary, Newtonian electrolyte solutions. All of these simplifications are similar to those invoked in many other circuit-based analyses of microfluidic networks (Qiao and Aluru 2002; Xuan and Li 2004; Chatterjee and Aluru 2005; Berli 2007, 2008; Biscombe et al. 2011).

\section{Results and discussion}

We use the term 'error' to mean the error in TCM predictions relative to the more rigorous ICM predictions, that is,

$$
\begin{aligned}
& \eta_{p}=\left|\frac{\Delta p_{\mathrm{t}, \mathrm{TCM}}-\Delta p_{\mathrm{t}, \mathrm{ICM}}}{\Delta p_{\mathrm{t}, \mathrm{ICM}}}\right|, \\
& \eta_{\phi}=\left|\frac{\Delta \phi_{\mathrm{t}, \mathrm{TCM}}-\Delta \phi_{\mathrm{t}, \mathrm{ICM}}}{\Delta \phi_{\mathrm{t}, \mathrm{ICM}}}\right| .
\end{aligned}
$$

For electroviscous flow $(I=0)$, we previously showed that these errors are independent of flow rate (Biscombe et al. 2011). For mixed pressure-driven/electro-osmotic flow with $I \neq 0$, however, $\eta_{p}$ and $\eta_{\phi}$ both depend on $Q$ and $I$; these dependences can be reduced to a dependence on the single parameter $I / Q$ by noting that

$$
\begin{aligned}
& \frac{\Delta p}{Q}=\frac{I_{\mathrm{s}}}{G_{\mathrm{e}}}\left(\frac{L_{22}}{L_{21}}-\frac{I}{Q}\right), \\
& \frac{\Delta \phi}{Q}=-\frac{1}{G_{\mathrm{e}}}\left(I_{\mathrm{s}}+\frac{I}{Q}\right)
\end{aligned}
$$

(obtained by rearranging Eq. (2)). Here $I_{\mathrm{s}}=(I / Q)_{\Delta \phi=0}=L_{21} / L_{11}$ is the streaming current per unit flow rate and $G_{\mathrm{e}}=(I / \Delta \phi)_{Q=0}=\left(L_{11} L_{22}-L_{12} L_{21}\right) / L_{11}$ is the electrical conductance at zero net flow rate. Similarly, $L_{21}=(I / \Delta p)_{\Delta \phi=0}$ is the streaming current per unit pressure 
difference (also known as the streaming conductance (van der Heyden et al. 2005)) and $L_{22}=(I / \Delta \phi)_{\Delta p=0}$ is the electrical conductance at zero pressure difference. Substituting Eqs. (9) and (10) for each channel into Eqs. (7) and (8), the $Q$ in the denominator on the left-hand sides of Eqs. (9) and (10) cancels out. It is therefore possible to evaluate $\eta_{p}$ and $\eta_{\phi}$ by specifying the value of the flux ratio $I / Q$ (units $\mathrm{Cm}^{-3}$ ) alone rather than separately specifying both $I$ and $Q$.

\subsection{Base case}

Fig. 2 shows error contours for TCM predictions of the total pressure and potential differences occurring along the cylindrical contraction-expansion shown in Fig. 1. Qualitatively, $\eta_{p}$ and $\eta_{\phi}$ behave similarly throughout the entire parameter space considered (this trend is replicated for all of our results). For a fixed value of $-I / Q$, the maximum values of $\eta_{p}$ and $\eta_{\phi}$ (denoted by $\eta_{p, \max }$ and $\eta_{\phi, \max }$ respectively) occur at intermediate concentrations $\left(n_{0}^{\text {(in) }} \approx 10^{-3} \mathrm{M}\right)$; these concentrations increase gradually as the magnitude of $-I / Q$ increases. Remarkably, $\eta_{p}$ and $\eta_{\phi}$ deviate little from their values for the electroviscous case $(-I / Q=0)$ until $-I / Q$ exceeds about $10^{4} \mathrm{Cm}^{-3}$. When $-I / Q<10^{4} \mathrm{Cm}^{-3}, \eta_{p, \text { max }}<0.02$ and $\eta_{\phi, \text { max }}<0.07$ always, and so the TCM yields similar predictions to the ICM. For larger values of $-I / Q$, however, the maximum errors quickly increase: $\eta_{p}$ and $\eta_{\phi}$ both exceed 1 (i.e. more than $100 \%$ error) for inlet concentrations around $10^{-3} \mathrm{M}$ when $-I / Q=10^{7} \mathrm{Cm}^{-3}$, while for $-I / Q=10^{10} \mathrm{Cm}^{-3}, \eta_{p}$ and $\eta_{\phi}$ both exceed $1000 \%$ over a significant concentration range.

To provide a more tangible picture of the significance of the above results, Fig. 3 illustrates the dimensional pressure and potential differences predicted to occur for an inlet concentration $n_{0}^{\text {(in) }}$ of $10^{-3} \mathrm{M}$ and a flow rate $Q$ of $100 \mathrm{als}^{-1}\left(10^{-19} \mathrm{~m}^{3} \mathrm{~s}^{-1}\right.$; this yields an average residence time in the contraction of about $400 \mathrm{~s}$, which is comparable to the expected residence time for the experiments of van der Heyden et al. (2005) with a $70 \mathrm{~nm}$ high rectangular nanochannel at an applied pressure difference of $0.1 \mathrm{MPa}$ ). For the case with $-I / Q=10^{9} \mathrm{Cm}^{-3}$ (i.e. $-I=100 \mathrm{pA}$ ), the ICM predicts total pressure and potential changes of $\Delta p_{\mathrm{t}, \mathrm{ICM}}=-5.17 \mathrm{MPa}$ and $\Delta \phi_{\mathrm{t}, \mathrm{ICM}}=61.4 \mathrm{~V}$. The TCM, in contrast, yields $\Delta p_{\mathrm{t}, \mathrm{TCM}}=-148 \mathrm{MPa}$ and $\Delta \phi_{\mathrm{t}, \mathrm{TCM}}=657 \mathrm{~V}$, both of which exceed the more rigorous ICM predictions by over an order of magnitude. 

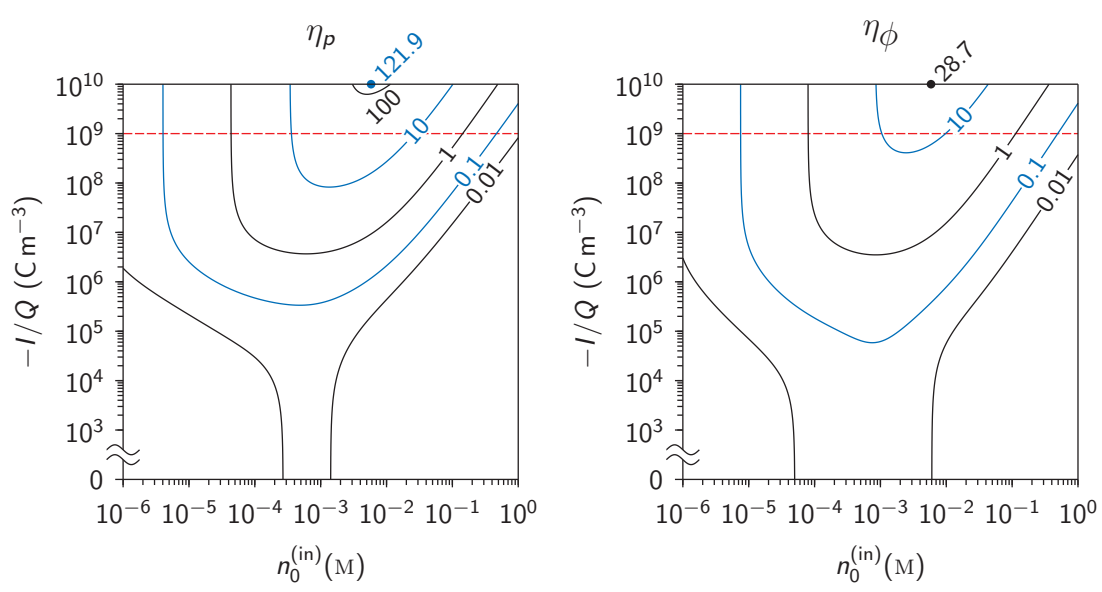

Fig. 2 TCM pressure and potential errors, $\eta_{p}$ (left pane) and $\eta_{\phi}$ (right pane) respectively, for the cylindrical base case shown in Fig. 1. The dashed line indicates the base value of $-I / Q=10^{9} \mathrm{Cm}^{-3}$ that we consider throughout this section.
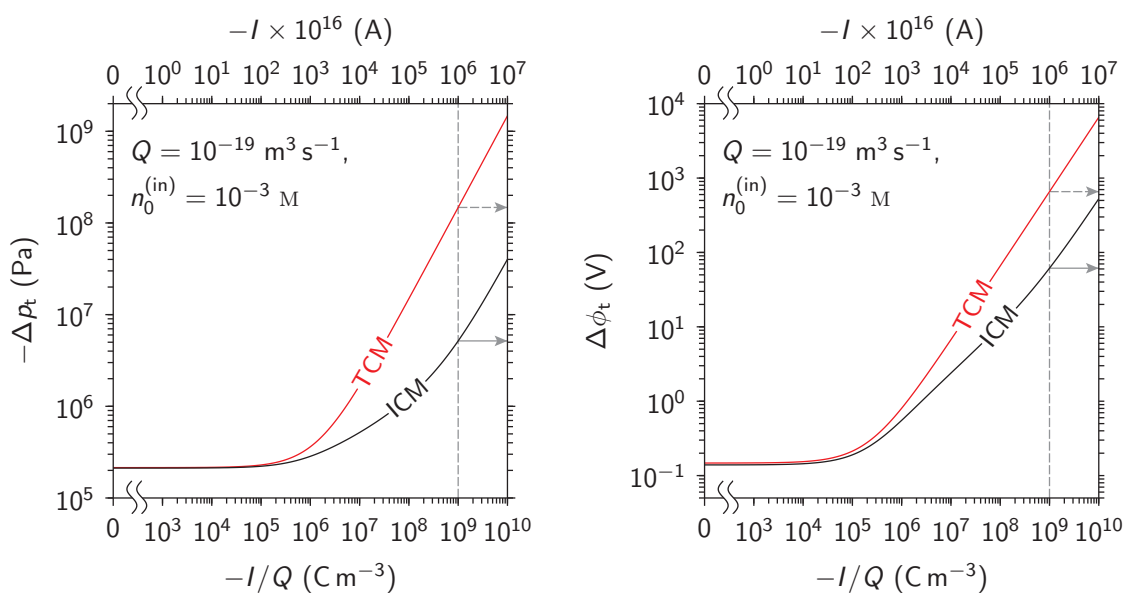

Fig. 3 Dimensional pressure and potential differences, $\Delta p_{\mathrm{t}}$ (left pane) and $\Delta \phi_{\mathrm{t}}$ (right pane) respectively, predicted for the base geometry with a flow rate of $10^{-19} \mathrm{~m}^{3} \mathrm{~s}^{-1}$ and an inlet concentration of $10^{-3} \mathrm{M}$. When the total current is $-10^{-10} \mathrm{~A}\left(-I / Q=10^{9} \mathrm{Cm}^{-3}\right)$, the TCM predictions exceed the ICM predictions by over an order of magnitude.

\subsection{Effect of changing to thin Cartesian slit geometry}

We also investigated a thin slit analogue of the base cylindrical geometry. The slit channels have the same dimensions as given in Table 1, but here $a$ represents the half-width of the channels. The channels are assumed to have a large (mathematically, infinite) aspect ratio. The results (not shown) closely resemble the results for the cylindrical geometry (shown in Fig. 2). 
$\eta_{p}$

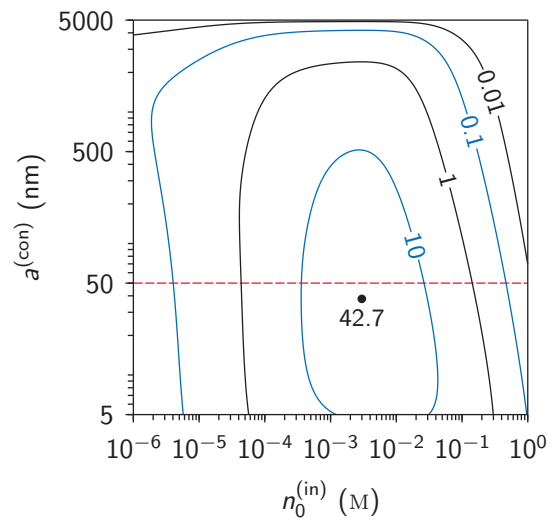

$\eta_{\phi}$

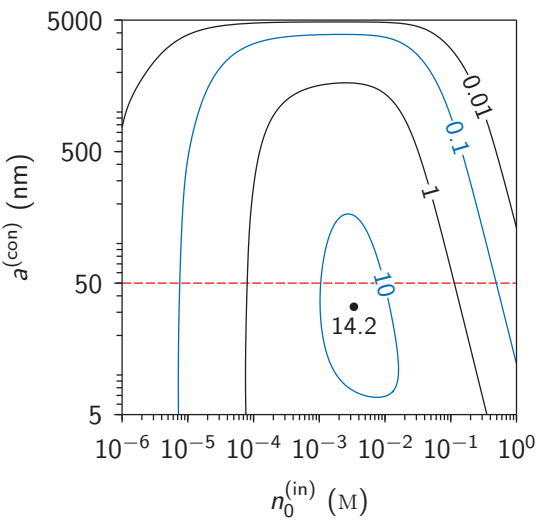

Fig. 4 TCM pressure and potential errors, $\eta_{p}$ (left pane) and $\eta_{\phi}$ (right pane) respectively, for different contraction radii. Here $-I / Q=10^{9} \mathrm{Cm}^{-3}$ and $\sigma_{\mathrm{d}}=-25 \mathrm{mCm}^{-2}$. The dashed line indicates the base contraction radius of $50 \mathrm{~nm}$.

Again, the errors are largely insensitive to $-I / Q$ for $-I / Q$ values of up to about $10^{4} \mathrm{Cm}^{-3}$, and as before the maximum error for a fixed value of $-I / Q$ occurs at an intermediate inlet concentration. The global maximum errors occur at the maximum value of $-I / Q$ considered $\left(10^{10} \mathrm{Cm}^{-3}\right)$ and are $\eta_{p, \max }=133.2$ and $\eta_{\phi, \text { max }}=16.1$; these are of comparable magnitude to the cylindrical case.

\subsection{Effect of contraction radius}

Fig. 4 illustrates the effect of altering the contraction radius. The smallest contraction radius we consider is $5 \mathrm{~nm}$, which is close to the accepted lower limit of validity of the continuum approximation (Daiguji 2010; Sparreboom et al. 2010) upon which the TCM and ICM are based. At the other extreme, we consider a contraction radius of $5 \mu \mathrm{m}$, that is, a system in which the 'contraction' is the same size as the inlet and outlet channels. As expected, $\eta_{p}$ and $\eta_{\phi}$ both approach zero as $a^{(\mathrm{con})}$ approaches $a^{\text {(in) }}\left(\right.$ since $n_{0}=n_{0}^{(\text {in) }}$ everywhere in that case). The largest errors occur at moderate concentrations and contraction radii close to the base value of $50 \mathrm{~nm}$. 
$\eta_{p}$

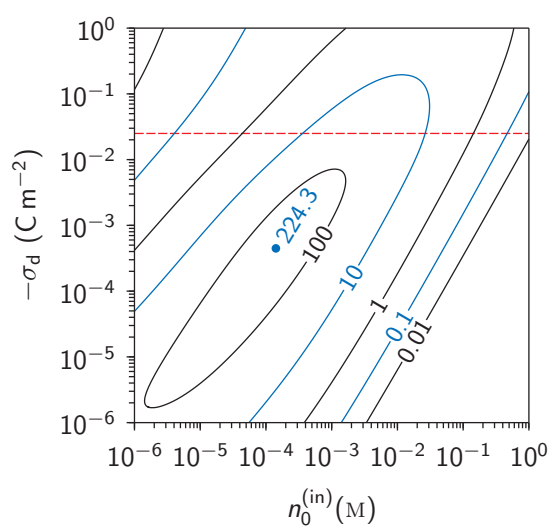

$\eta_{\phi}$

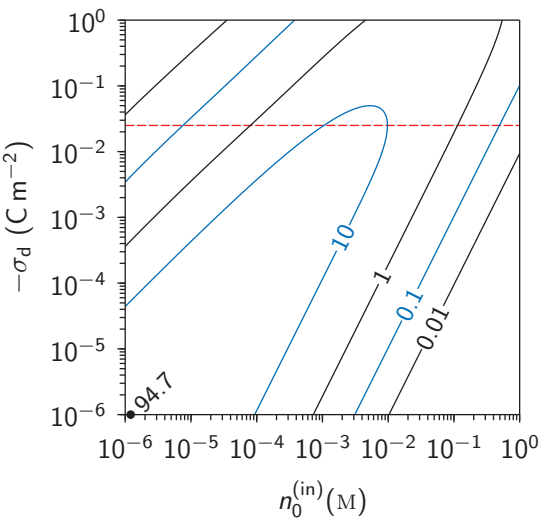

Fig. 5 TCM pressure and potential errors, $\eta_{p}$ (left pane) and $\eta_{\phi}$ (right pane) respectively, for different surface charge densities. Here $-I / Q=10^{9} \mathrm{Cm}^{-3}$ and $a^{(\mathrm{con})}=50 \mathrm{~nm}$. The dashed line corresponds to the base surface charge density of $\sigma_{\mathrm{d}}=-25 \mathrm{mCm}^{-2}$.

\subsection{Effect of surface charge density}

In reality, the surface charge of silica arises primarily due to deprotonation of silanol groups, and as such depends on the $p \mathrm{H}$ of the electrolyte solution (Behrens and Grier 2001). Values of $\sigma_{\mathrm{d}}$ for silica previously quoted in the literature vary (Stein et al. 2004; van der Heyden et al. 2005; Schoch and Renaud 2005; Andersen et al. 2011). Fig. 5 shows error contours for surface charge densities ranging from $-10^{-6} \mathrm{Cm}^{-2}$ to $-1 \mathrm{Cm}^{-2}$, with $-I / Q=10^{9} \mathrm{Cm}^{-3}$. The errors are largest at low to moderate surface charge densities (approximately $10^{-6} \mathrm{Cm}^{-2} \leq$ $\left.\sigma_{\mathrm{d}} \leq 10^{-3} \mathrm{Cm}^{-2}\right)$ and low to moderate inlet concentrations $\left(10^{-6} \mathrm{M} \leq n_{0}^{(\mathrm{in})} \leq 10^{-3} \mathrm{M}\right)$. We have previously shown that the errors for electroviscous flow through the same channel network behave similarly (Biscombe et al. 2011), but in that case the errors (global maxima $\eta_{p, \max }=0.0250$ and $\eta_{\phi, \max }=0.167$ ) are much smaller than those seen here (global maxima $\eta_{p, \max }=224.3$ and $\left.\eta_{\phi, \max }=94.7\right)$.

3.5 The influence of $n_{0}$

It is possible to have $\Delta p_{\mathrm{t}, \mathrm{ICM}} \neq \Delta p_{\mathrm{t}, \mathrm{TCM}}$ and $\Delta \phi_{\mathrm{t}, \mathrm{ICM}} \neq \Delta \phi_{\mathrm{t}, \mathrm{TCM}}$ for a given set of inlet conditions because the ICM and TCM rely on different values of the reference ion concentration in the contraction, that is, $n_{0, \mathrm{ICM}}^{(\mathrm{con})} \neq n_{0, \mathrm{TCM}}^{\text {(con) }}$ (recall that $n_{0, \mathrm{TCM}}^{(\mathrm{con})}=n_{0}^{(\mathrm{in})}$ ). Fig. 6 shows how $n_{0, \mathrm{ICM}}^{\text {(con) }}$ varies with $n_{0}^{(\text {in) }}$ as the parameters $-I / Q, \sigma_{\mathrm{d}}$, and $a^{(\mathrm{con})}$ are independently adjusted. 
In all cases we find that $n_{0, \mathrm{ICM}}^{\text {(con) }} \geq n_{0}^{(\mathrm{in})}$, with equality occurring when $n_{0}^{(\mathrm{in})}$ is sufficiently large. The range of concentrations over which EDL overlap occurs in the contraction is marked in Fig. 6, based on the assumption that the EDLs overlap when the EDL potential at the centreline of the contraction is at least $1 \%$ of its value at the shear plane (i.e. when $\left.\psi(0) \geq 0.01 \psi\left(a^{(\mathrm{con})}\right)\right)$. We find that $n_{0, \text { ICM }}^{(\mathrm{con})}$ may differ from $n_{0}^{(\mathrm{in})}$ even when the EDLs do not overlap, contrary to widely held perceptions (see e.g. Wang et al. (2010)). This behaviour occurs because even when the EDLs are relatively thin, the fractional contribution of EDL fluxes to the ion currents may still be significant, particularly due to the enhanced counterion conductivity within the EDLs (relative to the neutral electrolyte conductivity).

Fig. 6(a) shows that $n_{0, \text { ICM }}^{\text {(con) }}$ is largely independent of the value of $-I / Q$ when $-I / Q<$ $10^{4} \mathrm{Cm}^{-3}$, consistent with our observations from Fig. 2 . For larger values of $-I / Q, n_{0, \text { ICM }}^{\text {(con) }}$ increases as $-I / Q$ increases at a fixed value of $n_{0}^{(\text {in) }}$. When $-I / Q=10^{10} \mathrm{Cm}^{-3}, n_{0, \text { ICM }}^{\text {(con) }}$ exceeds $n_{0}^{\text {(in) }}$ over the entire range of $n_{0}^{\text {(in) }}$ values considered. At even larger values of $-I / Q$ and high values of $n_{0}^{\text {(in) }}, n_{0, \text { ICM }}^{\text {(con) }}$ becomes sufficiently large to violate the dilute solution assumption, and when $-I / Q=10^{12} \mathrm{Cm}^{-3}, n_{0, \text { ICM }}^{\text {(con) }}$ (numerically) exceeds the solubility limit (4.75 $\mathrm{M}$ for $\mathrm{KCl}$ at $25^{\circ} \mathrm{C}$ (Haynes 2011)) when $n_{0}^{(\text {in) }}$ is large. The maximum value of $-I / Q$ considered here, $10^{10} \mathrm{Cm}^{-3}$, is therefore close to the maximum practical value for the particular channel/electrolyte system investigated.

Why do the largest errors $\eta_{p, \max }$ and $\eta_{\phi \text {,max }}$ occur at intermediate inlet concentrations? The answer is that $\eta_{p}$ and $\eta_{\phi}$ both approach zero at the extreme ends of the concentration range. For large values of $n_{0}^{\text {(in) }}$, the errors become small because $n_{0, \mathrm{ICM}}^{\text {(con) }} \rightarrow n_{0, \mathrm{TCM}}^{\text {(con) }}$ as shown in Fig. 6, so that the ICM and TCM become essentially identical to each other. (Physically, this occurs because fluxes in the electroneutral bulk fluid make the dominant contribution to the ion currents when the EDLs are very thin, so that the TCM effectively conserves ion charge at high concentrations.) For small values of $n_{0}^{(\text {in) }}$, on the other hand, it is not immediately obvious why the predictions of the two models should agree, particularly since Fig. 6 clearly shows that $n_{0, \mathrm{ICM}}^{\text {(con) }}$ may be several orders of magnitude larger than $n_{0, \mathrm{TCM}}^{\text {(con) }}$. The explanation is that the streaming currents $L_{21}$ and $I_{\mathrm{s}}$ and the electrical conductances $L_{22}$ and $G_{\mathrm{e}}$ all become independent of $n_{0}$ (i.e. they approach constant values) in the dilute limit (Stein et al. 2004; van der Heyden et al. 2005; Schoch and Renaud 2005; Chang and Yossifon 2009), as illustrated in Fig. 7. These plateaux arise as a consequence of electroneutrality. The streaming current plateaux occur because the total charge present within the EDLs is constant (of equal magnitude but opposite sign to the wall charge), so that the rate of charge 

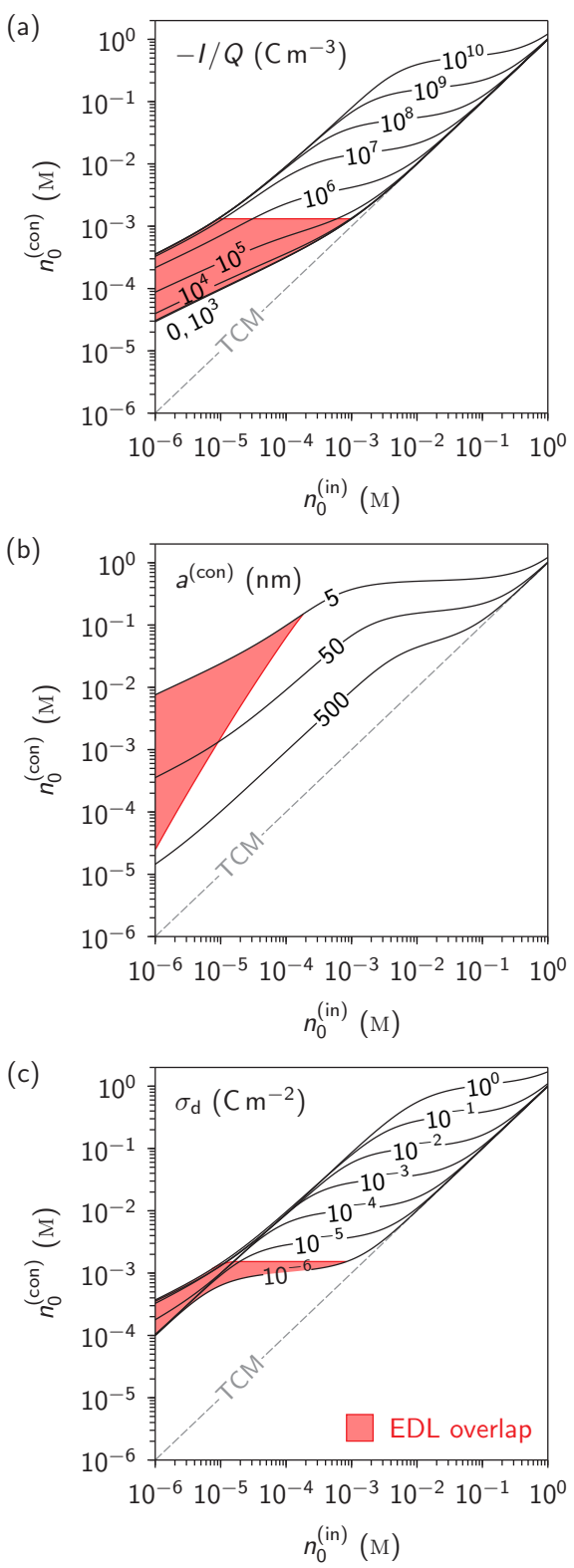

Fig. 6 ICM predictions of the geometric mean ion concentration in the contraction, $n_{0, \mathrm{ICM}}^{\text {(con) }}$ (a) Variable $-I / Q\left(\mathrm{Cm}^{-3}\right)$ with $\sigma_{\mathrm{d}}=-25 \mathrm{mCm}^{-2}$ and $a^{(\mathrm{con})}=50 \mathrm{~nm}$. (b) Variable contraction radius $a^{(\mathrm{con})}$ (nm) with $-I / Q=10^{9} \mathrm{Cm}^{-3}$ and $\sigma_{\mathrm{d}}=-25 \mathrm{mCm}^{-2}$. (c) Variable surface charge density $\sigma_{\mathrm{d}}\left(\mathrm{Cm}^{-2}\right)$ with $-I / Q=$ $10^{9} \mathrm{Cm}^{-3}$ and $a^{(\mathrm{con})}=50 \mathrm{~nm}$. The dashed lines show the TCM result for all parameter values $\left(n_{0, \mathrm{TCM}}^{(\mathrm{con})}=n_{0}^{(\mathrm{in})}\right)$. The shaded region indicates the range of concentrations over which EDL overlap occurs. 
advection (i.e. the streaming current, by definition) is limited by the fluid velocity within the EDLs. As $n_{0}$ is reduced, the EDLs expand towards the centre of the channel and the maximum velocity within the EDLs increases (at fixed flow rate), thereby increasing the rate of charge advection. Once the EDLs are strongly overlapped (as occurs at sufficiently low values of $n_{0}$ ), however, no further increase in the charge advection rate is possible. The electrical conductance plateaux occur because a finite minimum number of counterions must be present in order to neutralise the surface charge. When the EDLs overlap, the majority of ions in the channel are counterions and so the electrical conductance at low $n_{0}$ is dominated by counterion transport. All quantities on the right-hand sides of Eqs. (9) and (10) therefore approach constant values - and hence become insensitive to the value of $n_{0}^{(\text {con })}$ chosen-as $n_{0}^{(\text {in })} \rightarrow 0$.

For small values of the surface charge density (say $\sigma_{\mathrm{d}}<10^{-4} \mathrm{Cm}^{-2}$ ), Fig. 5 apparently shows that $\eta_{p, \max }$ and $\eta_{\phi, \text { max }}$ occur at low (rather than intermediate) concentrations ( $n_{0}^{(\mathrm{in})}<$ $\left.10^{-4} \mathrm{M}\right)$. In fact the plateau behaviour described above still occurs, but an inlet concentration of $10^{-6} \mathrm{M}$ (the minimum value of $n_{0}^{(\text {in) }}$ considered here) is not sufficiently low for the effect to become apparent: the maximum concentration at which the electrical conductance plateaux are observed decreases as the surface charge density is reduced (Schoch and Renaud 2005).

\section{Conclusions}

The performance of two different electrokinetic circuit models was evaluated:

1.The TCM (Xuan and Li 2004; Berli 2007) is based on an equilibrium species concentration constraint at circuit nodes (Eq. (5)) that may not be physically realistic in non-stationary (flowing) systems (Biscombe et al. 2012). The primary advantage of the TCM is that it is linear and therefore comparatively straightforward to solve.

2.The ICM (Harvie et al. 2012; Biscombe et al. 2012) explicitly accounts for ion fluxes across circuit nodes, thereby conserving species exactly. The downside is that the ICM is non-linear and requires an iterative numerical solution procedure.

Our results indicate that using the TCM may lead to gross errors (up to several thousand percent) in the prediction of the total pressure and potential differences for mixed pressuredriven/electro-osmotic flow through a practical contraction-expansion network. We find that the TCM does provide reasonably accurate predictions when the flux ratio $-I / Q$ is 

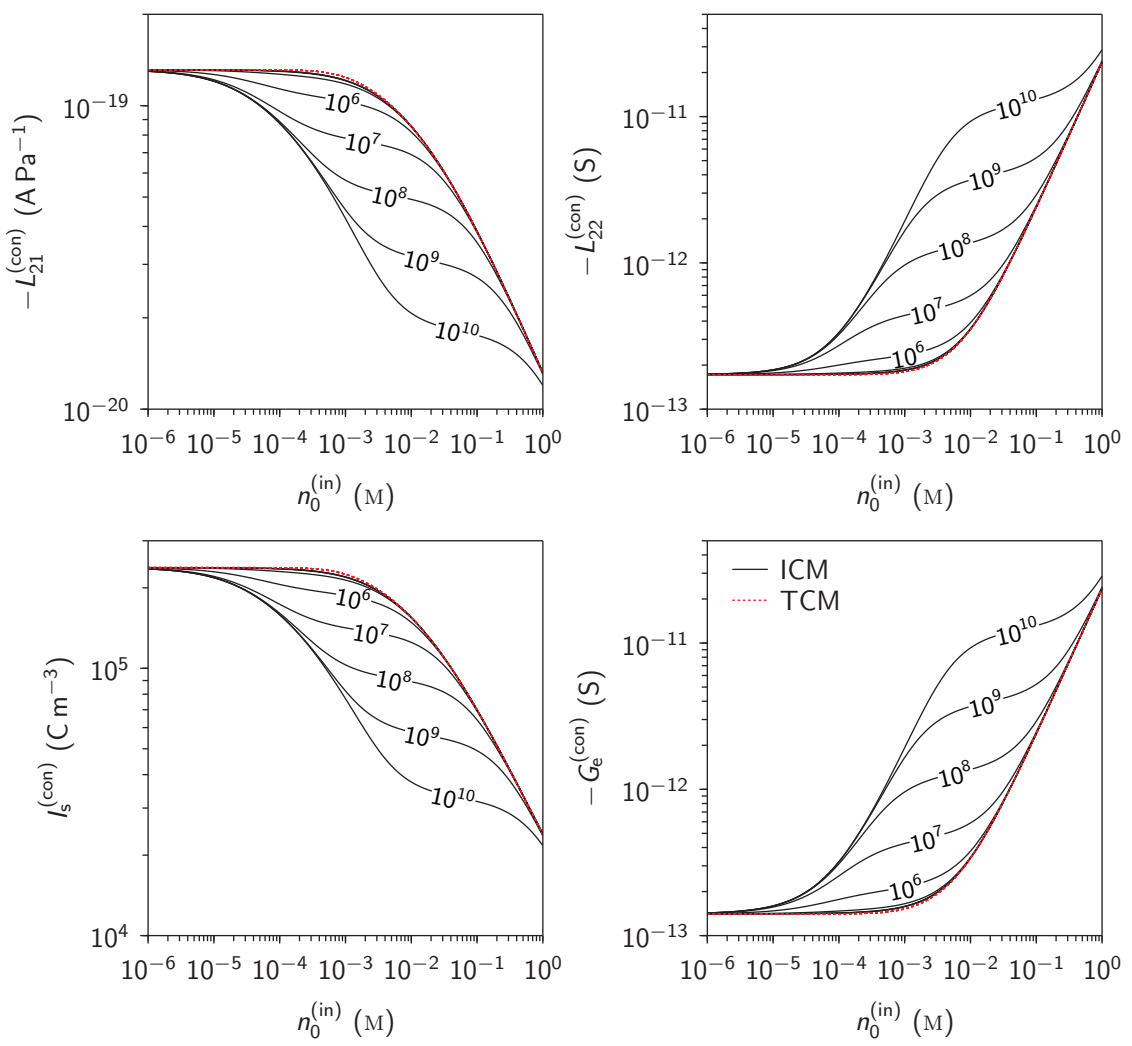

Fig. 7 ICM predictions (solid curves) of the streaming currents $L_{21}$ and $I_{\mathrm{s}}$ and electrical conductances $L_{22}$ and $G_{\mathrm{e}}$ with respect to the inlet concentration $n_{0}^{(\mathrm{in})}$ for $-I / Q$ values (labelled) between $0 \mathrm{Cm}^{-3}$ and $10^{10} \mathrm{Cm}^{-3}$ (curves for $-I / Q \leq 10^{5} \mathrm{Cm}^{-3}$ are not labelled because they are almost coincident with each other and with the TCM curve). TCM predictions (which do not depend on $-I / Q$ ) are shown as dashed curves. In all cases $\sigma_{\mathrm{d}}=-25 \mathrm{mCm}^{-2}$ and $a^{(\mathrm{con})}=50 \mathrm{~nm}$.

small or when the inlet concentration is either sufficiently low or sufficiently high. A precise quantification of these bounds requires detailed analysis of the specific system of interest, however, which largely undermines the use of the TCM as a predictive design tool.

We have demonstrated that the geometric mean ion concentration within a particular channel need not be equal to the concentration within the reservoirs to which it is connected (either directly or indirectly), and thus that Eq. (5) (upon which the TCM is based) is not generally valid. Significantly, we have also shown that a simple dichotomy based on EDL overlap alone does not provide a reliable basis for adopting the TCM: the deciding factor is rather the fractional contribution of fluxes within the EDLs to the total flux. 
Acknowledgements This research was partly supported by an Australian Research Council Discovery Grant.

\section{Appendix: Definitions of the conductances}

For a symmetric, binary electrolyte with $z=z_{+}=-z_{-}$, the conductances for slit $(\delta=0)$ and cylindrical $(\delta=1)$ microchannels are defined as

$$
\begin{gathered}
L_{11}=-\frac{1}{3}\left(\frac{3}{8}\right)^{\delta} \frac{a^{2} A}{\mu l}, \\
L_{12}=L_{21}=\frac{z e n_{0} a^{2} A}{\mu l} \mathscr{G}_{1}, \\
L_{22}=-\frac{\left(z e n_{0} a\right)^{2} A}{\mu l}\left[\mathscr{G}_{3,+}+\mathscr{G}_{3,-}\right. \\
\left.+\frac{\mu}{a^{2} n_{0} k T}\left(D_{+} \mathscr{G}_{4,+}+D_{-} \mathscr{G}_{4,-}\right)\right],
\end{gathered}
$$

where $A$ is the cross-sectional area. The $\mathscr{G}$ coefficients, which represent various integrals involving the transverse ion concentration profiles, are dimensionless constants that depend on $a, n_{0}, T, z, \varepsilon_{\mathrm{f}}$, and $\sigma_{\mathrm{d}}$. Harvie et al. (2012) present methods for evaluating the $\mathscr{G}$ coefficients.

\section{References}

Ajdari A (2004) Steady flows in networks of microfluidic channels: building on the analogy with electrical circuits. C R Phys 5:539-546

Andersen MB, Frey J, Pennathur S, Bruus H (2011) Surface-dependent chemical equilibrium constants and capacitances for bare and 3-cyanopropyldimethylchlorosilane coated silica nanochannels. J Colloid Interface Sci 353:301-310

Behrens SH, Grier DG (2001) The charge of glass and silica surfaces. J Chem Phys 115(14):6716-6721

Berli CLA (2007) Theoretical modelling of electrokinetic flow in microchannel networks. Colloids Surf, A 301:271-280

Berli CLA (2008) Equivalent circuit modeling of electrokinetically driven analytical microsystems. Microfluid Nanofluid 4:391-399

Berry JD, Davidson MR, Bharti RP, Harvie DJE (2011) Effect of wall permittivity on electroviscous flow through a contraction. Biomicrofluidics 5(4):044102

Bharti RP, Harvie DJE, Davidson MR (2008) Steady flow of ionic liquid through a cylindrical microfluidic contraction-expansion pipe: electroviscous effects and pressure drop. Chem Eng Sci 63:3593-3604

Biscombe CJC, Davidson MR, Harvie DJE (2011) Comparative evaluation of microfluidic circuit model performance for electroviscous flow. ANZIAM J 52:C447-C462

Biscombe CJC, Davidson MR, Harvie DJE (2012) Microfluidic circuit analysis II: Implications of ion conservation for microchannels connected in series. J Colloid Interface Sci 365:16-27 
Bousse L, Cohen C, Nikiforov T, Chow A, Kopf-Sill AR, Dubrow R, Parce JW (2000) Electrokinetically controlled microfluidic analysis systems. Annu Rev Biophys Biomol Struct 29:155-181

Boy DA, Gibou F, Pennathur S (2008) Simulation tools for lab on a chip research: advantages, challenges, and thoughts for the future. Lab Chip 8:1424-1431

Bruin GJM (2000) Recent developments in electrokinetically driven analysis on microfabricated devices. Electrophoresis 21:3931-3951

Brunet E, Ajdari A (2004) Generalized Onsager relations for electrokinetic effects in anisotropic and heterogeneous geometries. Phys Rev E 69:016306

Chang HC, Yossifon G (2009) Understanding electrokinetics at the nanoscale: a perspective. Biomicrofluidics 3(1):012001

Chatterjee AN, Aluru NR (2005) Combined circuit/device modeling and simulation of integrated microfluidic systems. J Microelectromech Syst 14(1):81-95

Daiguji H (2010) Ion transport in nanofluidic channels. Chem Soc Rev 39(3):901-911

Davidson MR, Harvie DJE (2007) Electroviscous effects in low Reynolds number liquid flow through a slit-like microfluidic contraction. Chem Eng Sci 62:4229-4240

Erickson D (2005) Towards numerical prototyping of labs-on-chip: modeling for integrated microfluidic devices. Microfluid Nanofluid 1:301-318

Gillespie D, Khair AS, Bardhan JP, Pennathur S (2011) Efficiently accounting for ion correlations in electrokinetic nanofluidic devices using density functional theory. J Colloid Interface Sci 359:520-529

Harvie DJE, Biscombe CJC, Davidson MR (2012) Microfluidic circuit analysis I: Ion current relationships for thin slits and pipes. J Colloid Interface Sci 365:1-15

Haynes WM (ed) (2011) CRC Handbook of Chemistry and Physics (Internet version), 91st edn. CRC Press/ Taylor and Francis, Boca Raton, Florida, USA

van der Heyden FHJ, Stein D, Dekker C (2005) Streaming currents in a single nanofluidic channel. Phys Rev Lett 95(11): 116104

Hughes BT, Berg JM, James DL, Ibraguimov A, Liu S, Temkin H (2008) One-dimensional axial simulation of electric double layer overlap effects in devices combining micro- and nanochannels. Microfluid Nanofluid 5:761-774

Hunter RJ (1981) Zeta Potential in Colloid Science: Principles and Applications. Academic Press, London

Jellema LC, Mey T, Koster S, Verpoorte E (2009) Charge-based particle separation in microfluidic devices using combined hydrodynamic and electrokinetic effects. Lab Chip 9:1914-1925

Jellema LJC, Markesteijn AP, Westerweel J, Verpoorte E (2010) Tunable hydrodynamic chromatography of microparticles localised in short microchannels. Anal Chem 82(10):4027-4035

Kim SJ, Wang YC, Lee JH, Jang H, Han J (2007) Concentration polarization and nonlinear electrokinetic flow near a nanofluidic channel. Phys Rev Lett 99(4):044501

Lettieri GL, Dodge A, Boer G, de Rooij NF, Verpoorte E (2003) A novel microfluidic concept for bioanalysis using freely moving beads trapped in recirculating flows. Lab Chip 3:34-39

Mani A, Zangle TA, Santiago JG (2009) On the propagation of concentration polarization from microchannelnanochannel interfaces. Part I: Analytical model and characteristic analysis. Langmuir 25(6):3898-3908 
Ninham BW, Parsegian VA (1971) Electrostatic potential between surfaces bearing ionizable groups in ionic equilibrium with physiologic saline solution. J Theor Biol 31:405-428

Onsager L (1931a) Reciprocal relations in irreversible processes. I. Phys Rev 37:405-426

Onsager L (1931b) Reciprocal relations in irreversible processes. II. Phys Rev 38:2265-2279

Park SY, Russo CJ, Branton D, Stone HA (2006) Eddies in a bottleneck: an arbitrary Debye length theory for capillary electroosmosis. J Colloid Interface Sci 297:832-839

Plecis A, Schoch RB, Renaud P (2005) Ionic transport phenomena in nanofluidics: experimental and theoretical study of the exclusion-enrichment effect on a chip. Nano Lett 5(6):1147-1155

Postler T, Slouka Z, Svoboda M, Přibyl M, Šnita D (2008) Parametrical studies of electroosmotic transport characteristics in submicrometer channels. J Colloid Interface Sci 320:321-332

Pu Q, Yun J, Temkin H, Liu S (2004) Ion-enrichment and ion-depletion effect of nanochannel structures. Nano Lett 4(6):1099-1103

Qiao R, Aluru NR (2002) A compact model for electroosmotic flows in microfluidic devices. J Micromech Microeng 12:625-635

Ramirez JC, Conlisk AT (2006) Formation of vortices near abrupt nano-channel height changes in electroosmotic flow of aqueous solutions. Biomed Microdevices 8:325-330

Schoch RB, Renaud P (2005) Ion transport through nanoslits dominated by the effective surface charge. Appl Phys Lett 86(25):253111

Sørensen TS, Koefoed J (1974) Electrokinetic effects in charged capillary tubes. J Chem Soc, Faraday Trans 2 70:665-675

Sparreboom W, van den Berg A, Eijkel JCT (2010) Transport in nanofluidic systems: a review of theory and applications. New J Phys 12:015004

Stein D, Kruithof M, Dekker C (2004) Surface-charge-governed ion transport in nanofluidic channels. Phys Rev Lett 93(3):035901

Verpoorte E (2002) Microfluidic chips for clinical and forensic analysis. Electrophoresis 23:677-712

Wachutka G (1995) Tailored modeling: a way to the 'virtual microtransducer fab'? Sens Actuators, A 47:603612

Wang M, Kang Q, Ben-Naim E (2010) Modeling of electrokinetic transport in silica nanofluidic channels. Anal Chim Acta 664:158-164

Wang Y, Pant K, Chen Z, Wang G, Diffey WF, Ashley P, Sundaram S (2009) Numerical analysis of electrokinetic transport in micro-nanofluidic interconnect preconcentrator in hydrodynamic flow. Microfluid Nanofluid 7:683-696

Wang YC, Stevens AL, Han J (2005) Million-fold preconcentration of proteins and peptides by nanofluidic filter. Anal Chem 77(14):4293-4299

Xuan X, Li D (2004) Analysis of electrokinetic flow in microfluidic networks. J Micromech Microeng $14: 290-298$

Zangle TA, Mani A, Santiago JG (2009) On the propagation of concentration polarization from microchannelnanochannel interfaces. Part II: Numerical and experimental study. Langmuir 25(6):3909-3916 


\section{University Library}

\section{- M M I N E R VA A gateway to Melbourne's research publications}

Minerva Access is the Institutional Repository of The University of Melbourne

Author/s:

Biscombe, CJC;Davidson, MR;Harvie, DJE

Title:

Electrokinetic flow in connected channels: a comparison of two circuit models

Date:

2012-09-01

Citation:

Biscombe, C. J. C., Davidson, M. R. \& Harvie, D. J. E. (2012). Electrokinetic flow in connected channels: a comparison of two circuit models. MICROFLUIDICS AND NANOFLUIDICS, 13 (3), pp.481-490. https://doi.org/10.1007/s10404-012-0996-5.

Persistent Link:

http://hdl.handle.net/11343/283099 